\title{
Analytical solutions of linear inhomogeneous fractional differential equation with continuous variable coefficients
}

Sunae Pak', Huichol Choi ${ }^{1}$, Kinam $\operatorname{Sin}^{1 *}$ and Kwang Ri ${ }^{2}$

"Correspondence:

18348668513@163.com

1 Faculty of Mathematics, Kim II Sung

University, Pyongyang, Democratic

People's Republic of Korea

Full list of author information is

available at the end of the article

\section{Springer}

\begin{abstract}
This paper proposes a series-representations for the solution of initial value problems of linear inhomogeneous fractional differential equation with continuous variable coefficients. It is proved that the solution of the problem is determined by adding the solution of the inhomogeneous differential equations with the homogeneous initial conditions to the linear combination of the canonical fundamental system of solution for corresponding homogeneous fractional differential equation and the inhomogeneous initial values. The effectiveness of the theoretical analysis is illustrated with two examples.
\end{abstract}

Keywords: Linear inhomogeneous equation; Fractional differential equation; Continuous variable coefficient

\section{Introduction}

The fractional modelings have aroused much attention in the fields of both engineering and mathematics due to their significant applications in diverse scientific areas such as electromagnetism [1], behaviors of physical phenomena [2], signal processing [3], and control engineering [4]. A lot of theoretical research has been carried on the existence and uniqueness of solution of fractional differential equations (FDEs) over the last years [5-10]. Currently, methods for solving FDEs with initial conditions can be classified into two classes, namely, approximative method and analytical methods. Typical approximative methods include the operational matrix method based on orthogonal functions, the predictor-corrector method, fractional Euler method, and so on ([11-16]). The most practical analytical methods are the Adomian decomposition method, the homotopy analysis method, the homotopy perturbation method, the Laplace transform method, and the variational iteration method ([17-21]).

In [22], a solution of general linear inhomogeneous fractional differential equations with constant coefficients has been obtained by using the Adomian decomposition method and one proved that this solution is equal to the solution represented by Green's function. A theory on the system of linear inhomogeneous fractional differential equation has been studied, and the solution was represented in terms of the Green function for the case

(c) The Author(s) 2019. This article is distributed under the terms of the Creative Commons Attribution 4.0 International License (http://creativecommons.org/licenses/by/4.0/), which permits unrestricted use, distribution, and reproduction in any medium, provided you give appropriate credit to the original author(s) and the source, provide a link to the Creative Commons license, and indicate if changes were made. 
of constant matrix coefficients in [23]. In [24], a power series solution method for some linear fractional differential equations with continuous variable coefficients has been presented. In [25], a generalization of Duhamel's method for one-term fractional differential equations with constant coefficients has been proposed in the case when the classical Duhamel's principle does not hold true for differential equations with Caputo fractional derivative. A fractional power series method has been introduced for the solution of fractional heat-like equations with variable coefficients in [26].

Our work proposes series-representations for the solution of linear inhomogeneous fractional differential equation with continuous variable coefficients and inhomogeneous initial conditions. The fractional derivative is of Caputo type in the proposed problem.

The remainder of the paper is organized as follows. In Sect. 2, some definitions of fractional calculus are introduced. Section 3 gives series-representations of solutions for initial value problems of linear inhomogeneous fractional differential equation with continuous variable coefficients. In Sect. 4, the effectiveness of the proposed theory is illustrated with two examples. Finally, the conclusion to our work is summarized in Sect. 5.

\section{Preliminaries}

Definition 2.1 Let $\mathbf{R}=(-\infty,+\infty)$ and $\mathbf{R}_{+}=(0,+\infty)$. We denote the space of functions $f$ by $C_{r}^{n}[0, T]$, where $f$ satisfies $f:(0, T] \rightarrow \mathbf{R}(\forall T>0)$ and $t^{r} f^{(n)}(t) \in C[0, T]$ for $0 \leq r<1$. In particular, denote $C_{r}^{0}[0, T]$ by $C_{r}[0, T]$.

Definition 2.2 ([27]) Let $\alpha \in \mathbf{R}_{+}, f \in C_{r}[0, T], 0 \leq r<1$. Then

$$
I_{0+}^{\alpha} f(t)=\frac{1}{\Gamma(\alpha)} \int_{0}^{t}(t-\tau)^{\alpha-1} f(\tau) d \tau, \quad t>0,
$$

is called a fractional integral of order $\alpha(\alpha>0)$ of the function $f$ in the sense of RiemannLiouville. In particular, we denote $I^{0} f(t)=f(t)$.

Definition 2.3 ([27]) Let $n-1<\alpha \leq n, n \in \mathbf{N}, I^{n-\alpha} f \in C_{\gamma}^{n}[0, T]$ and $0 \leq \gamma<1$. Then

$$
D_{0+}^{\alpha} f(t)=D^{n} I^{n-\alpha} f(t), \quad D^{n}=\frac{d^{n}}{d t^{n}},
$$

is called the fractional derivative of order $\alpha$ of the function $f$ in the sense of RiemannLiouville.

Definition 2.4 ([27]) Let $n-1<\alpha \leq n, n \in \mathbf{N}, I^{n-\alpha} f \in C_{r}^{n}[0, T], 0 \leq r<1$. Then

$$
{ }^{c} D_{0+}^{\alpha} f(t)=D_{0+}^{\alpha}\left[f(t)-\sum_{k=0}^{n-1} \frac{f^{(k)}(0)}{k !} t^{k}\right],
$$

is called the Caputo fractional derivative of order $\alpha$ of the function $f$.

Remark When $\alpha=n$, we have ${ }^{c} D_{0_{+}}^{\alpha} f(t)=D_{0_{+}}^{\alpha} f(t)=D^{n} f(t)$. 
Definition 2.5 Let $n \in \mathbf{N}$. We define the set

$$
A C^{n}[a, b]:=\left\{f:[a, b] \rightarrow \mathbf{R} \mid D^{n-1} f \in A C[a, b], D=\frac{d}{d t}\right\} .
$$

Here $A C^{1}[a, b]=A C[a, b]$ is the set of absolutely continuous functions on $[a, b]$.

Definition 2.6 We denote by $I^{\alpha}\left(L_{1}\right)$ the set of functions $f$ which are represented as an integral of order $\alpha>0$ of some integrable function $\varphi \in L_{1}(0, T)$, that is, $f=I^{\alpha} \varphi$.

\section{Main result}

Now we consider a linear inhomogeneous fractional differential equation with continuous variable coefficients

$$
{ }^{c} D_{0+}^{\alpha_{0}} y(t)+\sum_{i=1}^{m} a_{i}(t)^{c} D_{0+}^{\alpha_{i}} y(t)=g(t), \quad t \in[0, T]
$$

with the initial condition

$$
\left.D^{k} y(t)\right|_{t=+0}=b_{k} \in \mathbf{R}, \quad k=0,1, \ldots, n_{0}-1,
$$

where $\alpha_{0}, \alpha_{i} \in \mathbf{R}_{+}, i=1, \ldots, m$ satisfy $\alpha_{0}>0, \alpha_{0}>\alpha_{1}>\cdots>\alpha_{m} \geq 0$ and $n_{0}, n_{i}$ are nonnegative integers that satisfy $n_{0}-1<\alpha_{0} \leq n_{0}, n_{i}-1<\alpha_{i} \leq n_{i}, i=1, \ldots, m$.

We can write the corresponding homogeneous differential equation of Eq. (1) by

$$
{ }^{c} D_{0_{+}}^{\alpha_{0}} y(t)=-\sum_{i=1}^{m} a_{i}(t)^{c} D_{0_{+}}^{\alpha_{i}} y(t), \quad 0<t<T .
$$

In order to consider all possible cases for the homogeneous equation (3), we introduce the following index sets $\boldsymbol{H}_{j}$ provided by $\alpha_{i}, i=0,1, \ldots, m$ :

$$
\boldsymbol{H}_{j} \stackrel{d}{=}\left\{i: 0 \leq \alpha_{i} \leq j, i=1, \ldots, m\right\}, \quad j=0,1, \ldots, n_{0}-1 .
$$

Here, we set $h_{j}=\min \boldsymbol{H}_{j}$ if $\boldsymbol{H}_{j} \neq \varnothing$ (where $\varnothing$ is the empty set).

Remark Let $k \in \boldsymbol{H}_{j} \Rightarrow \alpha_{k} \leq j$ and $\boldsymbol{H}_{i} \subset \boldsymbol{H}_{j}(i<j)$. If $\boldsymbol{H}_{j} \neq \varnothing$, let $h_{j}=\min \boldsymbol{H}_{j}$ which is the smallest index of fractional orders of Eq. (3) that do not exceed $j$. Then it is evident that $m-h_{j}+1$ is the number of element of $\boldsymbol{H}_{j}$ and $h_{i} \geq h_{j}(i<j)$. Thus when $i<j, h_{i}-h_{j}$ represents the number of such order $\alpha_{k}$ that $i<\alpha_{k} \leq j$ and in particular, if $h_{i}=h_{j}$, then Eq. (3) has no such fractional orders $\alpha_{k}$ that $i<\alpha_{k} \leq j$.

Then there are the following possible cases:

Case 1. $\boldsymbol{H}_{0} \neq \varnothing$. In this case $\boldsymbol{H}_{j} \neq \varnothing, j=0,1, \ldots, n_{0}-1$.

Case 2. $n_{0} \geq 2$ and there exists a $j_{0} \in\left\{0,1, \ldots, n_{0}-2\right\}$ such that $\boldsymbol{H}_{j_{0}}=\varnothing$ and $\boldsymbol{H}_{j_{0}+1} \neq \varnothing$. In this case $\boldsymbol{H}_{j}=\varnothing, j=0,1, \ldots, j_{0}$ and $\boldsymbol{H}_{j} \neq \varnothing, j=j_{0}+1, \ldots, n_{0}-1$.

Case 3. $\boldsymbol{H}_{n_{0}-1}=\varnothing$. In this case $\boldsymbol{H}_{j}=\varnothing, j=0,1, \ldots, n_{0}-1$. 
Definition 3.1 The linear homogeneous fractional differential equation (3) is called of type 1,2,3, respectively, when Eq. (3) correspond to the cases 1, 2, 3 dependent on the patterns of distances between adjacent fractional orders.

Definition 3.2 A system of functions $y_{j}(t)\left(j=0,1, \ldots, n_{0}-1\right)$ is called a canonical fundamental system of solutions of the homogeneous equation (3) if it satisfies

$$
\begin{aligned}
& { }^{c} D_{0+}^{\alpha_{0}} y_{j}(t)=-\sum_{i=1}^{m} a_{i}(t)^{c} D_{0+}^{\alpha_{i}} y_{j}(t), \quad 0<t<T, \\
& \left.D^{k} y_{j}(t)\right|_{t=+0}= \begin{cases}1, & j=k, \\
0, & j \neq k, k, j=0,1, \ldots, n_{0}-1 .\end{cases}
\end{aligned}
$$

First, we consider the problem for solving the linear inhomogeneous fractional differential equations Eq. (1) with variable coefficients and the homogeneous initial condition

$$
\left.D^{k} y(t)\right|_{t=0+}=0, \quad k=0,1, \ldots, n_{0}-1 .
$$

Lemma 3.1 Let $g(t), a_{i}(t) \in C[0, T], i=1, \ldots, m$. Then the initial value problem (1) and (4) has the unique solution $y(t) \in C^{\alpha_{0}, n_{0}-1}[0, T]$, which is the limit $y(t)=\lim _{l \rightarrow \infty} y^{l}(t)$ of the approximation sequence

$$
\begin{aligned}
& y^{0}(t)=I_{0+}^{\alpha_{0}} g(t), \\
& y^{l}(t)=y^{0}(t)-I_{0+}^{\alpha_{0}} \sum_{i=1}^{m} a_{i}(t)^{c} D_{0+}^{\alpha_{i}} y^{l-1}(t), \quad l=1,2, \ldots,
\end{aligned}
$$

where $C^{\alpha_{0}, n_{0}-1}[0, T]:=C_{0}^{\alpha_{0}, n_{0}-1}[0, T]=\left\{y(x) \in C^{n_{0}-1}[0, T],{ }^{c} D_{0+}^{\alpha_{0}} y \in C[0, T]\right\}$ and the norm is

$$
\|y\|_{C^{\alpha_{0}, n_{0}-1}[0, T]}=\sum_{k=0}^{n_{0}-1}\left\|y^{(k)}\right\|_{C[0, T]}+\left\|{ }^{c} D_{0+}^{\alpha_{0}} y\right\|_{C[0, T]} .
$$

Proof First we prove the uniqueness and existence of solution for the initial value problem (1) and (4). Let assume that $y(t) \in C^{\alpha_{0}, n_{0}-1}[0, T]$ satisfy Eqs. (1) and (4). Then if ${ }^{c} D_{0+}^{\alpha_{0}} y(t)=$ $z(t)$, we take ${ }^{c} D_{0_{+}}^{\alpha_{0}} y(t) \in C[0, T]$ and $z(t) \in C[0, T]$. We can rewrite as

$$
I_{0+}^{\alpha_{0} c} D_{0+}^{\alpha_{0}} y(t)=I_{0+}^{\alpha_{0}} z(t)
$$

Also the left of the above equation has been changed,

$$
I_{0+}^{\alpha_{0} c} D_{0+}^{\alpha_{0}} y(t)=y(t)-\sum_{j=0}^{n_{0}-1} D^{j} y(0) \Phi_{j+1}(t), \quad \Phi_{j+1}(t)=\frac{t^{j}}{j !} .
$$

By the homogeneous initial condition (4), we obtain

$$
I_{0+}^{\alpha_{0} c} D_{0+}^{\alpha_{0}} y(t)=y(t)
$$


Namely $y(t)=I_{0+}^{\alpha_{0}} z(t)$. Therefore ${ }^{c} D_{0+}^{\alpha_{i}} y(t)=I_{0+}^{\alpha_{0}-\alpha_{i}} z(t)$ and Eq. (1) changes to the integral equation

$$
z(t)+\sum_{i=1}^{m} a_{i}(t) I_{0+}^{\alpha_{0}-\alpha_{i}} z(t)=g(t)
$$

Thus, for the solution $y(t) \in C^{\alpha_{0}, n_{0}-1}[0, T]$ of Eqs. (1) and (4), ${ }^{c} D_{0+}^{\alpha_{0}} y(t)=z(t)$ satisfies the integral equation Eq. (7).

Conversely let assume that $z(t) \in C[0, T]$ is the solution of Eq. (7). Since $g(t) \in C[0, T]$,

$$
I_{0+}^{\alpha_{0}} z(t)+I_{0+}^{\alpha_{0}} \sum_{i=1}^{m} a_{i}(t) I_{0+}^{\alpha_{0}-\alpha_{i}} z(t)=I_{0+}^{\alpha_{0}} g(t) .
$$

Let $y(t):=I_{0+}^{\alpha_{0}} z(t)$. Then we have ${ }^{c} D_{0+}^{\alpha_{i}} y(t)=I_{0+}^{\alpha_{0}-\alpha_{i}} z(t)$ and

$$
y(t)+I_{0+}^{\alpha_{0}} \sum_{i=1}^{m} a_{i}(t)^{c} D_{0+}^{\alpha_{i}} y(t)=I_{0+}^{\alpha_{0}} g(t) .
$$

That is,

$$
{ }^{c} D_{0+}^{\alpha_{0}} y(t)+\sum_{i=1}^{m} a_{i}(t)^{c} D_{0+}^{\alpha_{i}} y(t)=g(t) .
$$

Also we get

$$
\left.D^{k} y(t)\right|_{t=+0}=\left.D^{k} I_{0+}^{\alpha_{0}} z(t)\right|_{t=+0}=\left.I_{0+}^{\alpha_{0}-k} z(t)\right|_{t=+0}=0, \quad\left(k=0,1, \ldots, n_{0}-1\right)
$$

Therefore for the solution $z(t) \in C[0, T]$ of integral equation Eq. (7), $I_{0+}^{\alpha_{0}} z(t)=y(t) \in$ $C^{\alpha_{0}, n_{0}-1}[0, T]$ satisfies the initial value problem (1) and (4).

Thus the uniqueness and existence of solution for Eqs. (1) and (4) are equivalent to the ones of integral equation Eq. (7).

Now let prove the uniqueness and existence of solution for integral equation (7). Equation (7) can be rewritten

$$
z(t)=g(t)-\sum_{i=1}^{m} a_{i}(t) I_{0+}^{\alpha_{0}-\alpha_{i}} z(t)
$$

We define the operator $T$ by $T z(t):=g(t)-\sum_{i=1}^{m} a_{i}(t) I_{0+}^{\alpha_{0}-\alpha_{i}} z(t)$. Then Eq. (8) is expressed $z(t)=T z(t)$, namely $T: C[0, T] \rightarrow C[0, T]$.

In $C[0, T]$, we use the $k$-norm $\|z\|_{k}=\max _{k} e^{-k t}|z(t)|$ which is equivalent to the maxnorm.

For $\forall t \in[0, T]$, by the fact that $I^{\alpha_{0}-\alpha_{i}} e^{k t} \leq \frac{e^{k t}}{k^{\alpha_{0}-\alpha_{i}}}$, the following expression is derived:

$$
\begin{aligned}
& \left|T z_{1}(t)-T z_{2}(t)\right| \\
& \quad=\left|-\sum_{i=1}^{m} a_{i}(t) I_{0+}^{\alpha_{0}-\alpha_{i}}\left(z_{1}(t)-z_{2}(t)\right)\right| \leq \sum_{i=1}^{m}\left\|a_{i}\right\|_{\max } I_{0+}^{\alpha_{0}-\alpha_{i}}\left|z_{1}(t)-z_{2}(t)\right|
\end{aligned}
$$




$$
\begin{aligned}
& =\sum_{i=1}^{m}\left\|a_{i}\right\|_{\max } I_{0+}^{\alpha_{0}-\alpha_{i}} e^{k t} e^{-k t}\left|z_{1}(t)-z_{2}(t)\right| \leq\left\|z_{1}-z_{2}\right\|_{k} \sum_{i=1}^{m}\left\|a_{i}\right\|_{\max } I_{0+}^{\alpha_{0}-\alpha_{i}} e^{k t} \\
& \leq\left\|z_{1}-z_{2}\right\|_{k} \sum_{i=1}^{m}\left\|a_{i}\right\|_{\max } \frac{e^{k t}}{k^{\alpha_{0}-\alpha_{i}}} .
\end{aligned}
$$

So we can obtain

$$
e^{-k t}\left|T z_{1}(t)-T z_{2}(t)\right| \leq\left(\sum_{i=1}^{m}\left\|a_{i}\right\|_{\max } \frac{1}{k^{\alpha_{0}-\alpha_{i}}}\right)\left\|z_{1}-z_{2}\right\|_{k} .
$$

If $w(k):=\sum_{i=1}^{m}\left\|a_{i}\right\|_{\max } \frac{1}{k^{\alpha}-\alpha_{i}}$, then $\exists k_{0} \in \mathbf{R}_{+} ; \forall k>k_{0}, w\left(k_{0}\right)<1, w_{0}:=w\left(k_{0}\right),\left\|T z_{1}-T z_{2}\right\|_{k_{0}} \leq$ $w_{0}\left\|z_{1}-z_{2}\right\|_{k_{0}}$, i.e. the operator $T: C[0, T] \rightarrow C[0, T]$ is a contractive operator which is relative to $\|\cdot\|_{k_{0}}$. By the equivalence of $\|\cdot\|_{\max }$ and $\|\cdot\|_{k_{0}}, T: C[0, T] \rightarrow C[0, T]$ is the contractive operator which is relative to $\|\cdot\|_{\max }$.

Therefore by using Banach fixed point theorem, the integral equation (7) has a unique solution in the sense of the norm $\|\cdot\|_{\max }$ and the sequence $\left\{z_{n}(t)\right\}$ which is constructed by $z_{n}(t)=g(t)-\sum_{i=1}^{m} a_{i}(t) I_{0+}^{\alpha_{0}-\alpha_{i}} z_{n-1}(t)$ converges in the sense of the norm $\|\cdot\|_{\max }$ in $C[0, T]$.

Next let prove the convergence of the approximative sequence (5) and (6). Since $g(t) \in$ $C[0, T]$, the sequence $\left\{z_{n}(t)\right\}$ constructed by the approximative expression

$$
\left\{\begin{array}{l}
z_{0}=g(t) \\
z_{n}=g(t)-\sum_{i=1}^{m} a_{i}(t) I_{0+}^{\alpha_{0}-\alpha_{i}} z_{n-1}(t)
\end{array}\right.
$$

convergent in the sense of $\|\cdot\|_{\max }$.

From Eq. (9),

$$
\left\{\begin{array}{l}
I_{0+}^{\alpha_{0}} z_{0}=I_{0+}^{\alpha_{0}} g(t) \\
I_{0+}^{\alpha_{0}} z_{n}=I_{0+}^{\alpha_{0}} g(t)-I_{0+}^{\alpha_{0}} \sum_{i=1}^{m} a_{i}(t) I_{0+}^{\alpha_{0}-\alpha_{i}} z_{n-1}(t)
\end{array}\right.
$$

and since $I_{0+}^{\alpha_{0}} z_{k}=y^{k}, I_{0+}^{\alpha_{0}-\alpha_{i}} z_{k-1}(t)={ }^{c} D_{0+}^{\alpha_{i}} y^{k}(t)$, we have

$$
\left\{\begin{array}{l}
y^{0}=I_{0+}^{\alpha_{0}} g(t) \\
y^{l}=y^{0}-I_{0+}^{\alpha_{0}} \sum_{i=1}^{m} a_{i}(t)^{c} D_{0+}^{\alpha_{i}} y^{l}(t)
\end{array}\right.
$$

i.e., Eqs. (5) and (6), where $y^{l} \in C^{\alpha_{0}, n_{0}-1}[0, T]$.

We prove the convergence of sequence $\left\{y^{l}\right\}$ in $C^{\alpha_{0}, n_{0}-1}[0, T]$. Since $y^{k}=I_{0+}^{\alpha_{0}} z_{k}$,

$$
\begin{aligned}
& { }^{c} D_{0+}^{\alpha_{0}} y^{k}=z_{k}, \\
& D^{l} y^{k}=I_{0+}^{\alpha_{0}-l} z_{k}, \quad l=0,1, \ldots, n_{0}-1,
\end{aligned}
$$

are obtained. So

$$
\begin{aligned}
& \left\|{ }^{c} D^{\alpha_{0}} y^{k}\right\|_{\max }=\left\|z_{k}\right\|_{\max }, \\
& \left\|D^{l} y^{k}\right\|_{\max } \leq \frac{T^{\alpha_{0}-l}}{\Gamma\left(\alpha_{0}-l+1\right)}\left\|z_{k}\right\|_{\max }, \quad l=0,1, \ldots, n_{0}-1,
\end{aligned}
$$


are satisfied. From the above two equations, we have

$$
\sum_{l=1}^{n_{0}-1}\left\|D^{l} y^{k}\right\|_{\max }+\left\|{ }^{c} D_{0+}^{\alpha_{0}} y^{k}\right\|_{\max } \leq\left(\sum_{l=1}^{n_{0}-1} \frac{T^{\alpha_{0}-l}}{\Gamma\left(\alpha_{0}-l+1\right)}+1\right)\left\|z_{k}\right\|_{\max } .
$$

Since $\left\{z_{k}\right\}$ converges, the sequence $\left\{y^{k}\right\}$ constructed by Eqs. (5) and (6) converges in $C^{\alpha_{0}, n_{0}-1}[0, T]$.

Lemma 3.2 Let $\gamma=n_{0}-\alpha_{0}(\in[0,1)), n_{0}=n_{1}, a_{i} \in C_{\gamma}^{1}[0, T]$ and $D_{0+}^{n_{0}-\alpha_{0}} a_{i} \in C[0, T], i=$ $1, \ldots, m$. Also let assume that $\boldsymbol{H}_{0} \neq \varnothing$, that is, Eq. (3) is of type 1. Then there exists the unique canonical fundamental system $y_{j}(t) \in C_{\gamma}^{n_{0}}[0, T],\left(j=0,1, \ldots, n_{0}-1\right)$ of the solution for Eq. (3) and it is written

$$
\begin{aligned}
y_{j}(t) & =\Phi_{j+1}(t)+\sum_{k=0}^{\infty}(-1)^{k+1} I_{0+}^{\alpha_{0}}\left[\sum_{i=1}^{m} a_{i}(t) I_{0+}^{\alpha_{0}-\alpha_{i}}\right]^{k} \sum_{i=h_{j}}^{m} a_{i}(t) \Phi_{j+1-\alpha_{i}}(t), \\
j & =0,1, \ldots, n_{0}-1,
\end{aligned}
$$

where

$$
\Phi_{j+1}(t)=\frac{t^{j}}{j !}
$$

Proof Let find the canonical system as the limit in $C_{\gamma}^{n_{0}}[0, T]$ of the approximation sequence

$$
\begin{aligned}
& y_{j}^{0}(t)=\Phi_{j+1}(t), \\
& y_{j}^{l+1}(t)=\Phi_{j+1}(t)-I_{0+}^{\alpha_{0}}\left[\sum_{i=1}^{m} a_{i}(t)^{c} D_{0+}^{\alpha_{i}} y_{j}^{l}(t)\right], \quad l=0,1,2, \ldots
\end{aligned}
$$

First, we will obtain $y_{0}(t)$. Let $j=0$ in Eq. (11), then $y_{0}^{0}(t)=\Phi_{1}(t)$ and from Eq. (12), we have

$$
y_{0}^{1}(t)=\Phi_{1}(t)-I_{0+}^{\alpha_{0}} \sum_{i=1}^{m} a_{i}(t)^{c} D_{0+}^{\alpha_{i}} y_{0}^{0}(t)=\Phi_{1}(t)-I_{0+}^{\alpha} \sum_{i=1}^{m} a_{i}(t)^{c} D_{0+}^{\alpha_{i}} \Phi_{1}(t) .
$$

Also

$$
{ }^{c} D_{0+}^{\alpha_{i}} \Phi_{1}(t)=D_{0+}^{\alpha_{i}}\left[\Phi_{1}(t)-\sum_{k=0}^{n_{i}-1} D^{k} \Phi_{1}(0) \Phi_{k+1}(t)\right], \quad i=1,2, \ldots, m .
$$

Since $H_{0} \neq \varnothing$, we have $h_{0}=\min H_{0}=m$. For $i=m, \alpha_{m}=n_{m}=0$,

$$
{ }^{c} D_{0+}^{\alpha_{i}} \Phi_{1}(t)=D_{0+}^{\alpha_{m}} \Phi_{1}(t)=\Phi_{1}(t) .
$$

If $i=1, \ldots, m-1$, then $\alpha_{i}>0$ and from $n_{i}-1<\alpha_{i} \leq n_{i}$, we have $n_{i} \geq 1$. Since

$$
D^{k} \Phi_{1}(0)= \begin{cases}1, & k=0 \\ 0, & k \neq 0\end{cases}
$$


in (14), then ${ }^{c} D_{0_{+}}^{\alpha_{i}} \Phi_{1}(t)=0$ for $i=1, \ldots, m-1$ and

$$
{ }^{c} D_{0+}^{\alpha_{i}} \Phi_{1}(t)= \begin{cases}\Phi_{1}(t), & i=h_{0}=m \\ 0, & i=1, \ldots, m-1\end{cases}
$$

Substituting this into Eq. (13), then the first approximation of $y_{0}(t)$ is given by

$$
y_{0}^{1}(t)=\Phi_{1}(t)-I_{0+}^{\alpha_{0}} a_{m}(t) \Phi_{1}(t)
$$

and since $h_{0}=m$ and $\alpha_{m}=0$, we can rewrite it as

$$
y_{0}^{1}(t)=\Phi_{1}(t)-I_{0+}^{\alpha_{0}} \sum_{i=h_{0}}^{m} a_{i}(t) \Phi_{1-\alpha_{i}}(t) .
$$

Thus we get the first term $(k=0)$ of Eq. (10) in the case of $j=0$.

Now let prove $y_{0}^{1}(t) \in C_{\gamma}^{n_{0}}[0, T]$. From Eq. (15), we have

$$
D^{n_{0}-1} y_{0}^{1}(t)=D^{n_{0}-1} \Phi_{1}(t)-D^{n_{0}-1} I_{0+}^{\alpha_{0}} a_{m}(t) \Phi_{1}(t)=-I_{0+}^{\alpha_{0}-n_{0}+1} a_{m}(t) \Phi_{1}(t) .
$$

From $a_{m}(t) \Phi_{1}(t)=a_{m}(t) \in C[0, T]$ and $\alpha_{0}-n_{0}+1>0, I_{0+}^{\alpha_{0}-n_{0}+1} a_{m}(t) \Phi_{1}(t) \in C[0, T]$ is found, that is,

$$
y_{0}^{1}(t) \in C^{n_{0}-1}[0, T]
$$

On the other hand, from $a_{m}(t) \Phi_{1}(t)=a_{m}(t) \in C_{\gamma}^{1}[0, T]$ and $\alpha_{0}-n_{0}+1>0$, we have $I_{0+}^{\alpha_{0}-n_{0}+1} a_{m}(t) \Phi_{1}(t) \in C_{\gamma}^{1}[0, T]$. Therefore

$$
D^{n_{0}} y_{0}^{1}(t)=D^{n_{0}} \Phi_{1}(t)-D^{n_{0}} I_{0+}^{\alpha_{0}} a_{m}(t) \Phi_{1}(t)=-D I_{0+}^{\alpha_{0}-n_{0}+1} a_{m}(t) \Phi_{1}(t) \in C_{\gamma}[0, T]
$$

Thus we proved $y_{0}^{1}(t) \in C_{\gamma}^{n_{0}}[0, T]$.

Next we consider the case of $j=0, l=1$ in Eq. (12) to find the second approximation of $y_{0}(t)$. We have

$$
\begin{aligned}
y_{0}^{2}(t) & =\Phi_{1}(t)-I_{0+}^{\alpha_{0}} \sum_{i=1}^{m} a_{i}(t)^{c} D_{0+}^{\alpha_{i}} y_{0}^{1}(t) \\
& =\Phi_{1}(t)-I_{0+}^{\alpha_{0}} \sum_{i=1}^{m} a_{i}(t)^{c} D_{0+}^{\alpha_{i}}\left[\Phi_{1}(t)-I_{0+}^{\alpha_{0}} \sum_{i=h_{0}}^{m} a_{i}(t) \Phi_{1-\alpha_{i}}(t)\right] \\
& =\Phi_{1}(t)-I_{0+}^{\alpha_{0}} \sum_{i=1}^{m} a_{i}(t)^{c} D_{0+}^{\alpha_{i}} \Phi_{1}(t)+I_{0+}^{\alpha_{0}} \sum_{i=1}^{m} a_{i}(t)^{c} D_{0+}^{\alpha_{i}} I_{0+}^{\alpha_{0}} \sum_{i=h_{0}}^{m} a_{i}(t) \Phi_{1-\alpha_{i}}(t) .
\end{aligned}
$$

Now let $f(t):=\sum_{i=h_{0}}^{m} a_{i}(t) \Phi_{1-\alpha_{i}}(t)$ and calculate ${ }^{c} D_{0+}^{\alpha_{i}} \alpha_{0+}^{\alpha_{0}} f(t)$. Then we get

$$
{ }^{c} D_{0+}^{\alpha_{i}} I_{0+}^{\alpha_{0}} f(t)=D_{0+}^{\alpha_{i}}\left[I_{0+}^{\alpha_{0}} f(t)-\sum_{k=0}^{n_{i}-1} D^{k} I_{0+}^{\alpha_{0}} f(t)\right]_{t=+0} \Phi_{k+1}(t) .
$$


Here since $k \leq n_{i}-1<\alpha_{i}<\alpha_{0}, i=1, \ldots, m$, we have $\alpha_{0}-k>0, k=0,1, \ldots, n_{i}-1$ and thus we can rewrite it as

$$
D^{k} I_{0+}^{\alpha_{0}} f(t)=D^{k} I^{k} I_{0+}^{\alpha_{0}-k} f(t)=I_{0+}^{\alpha_{0}-k} f(t)
$$

Since $f(t) \in C[0, T]$, we have

$$
\left.D^{k} I_{0+}^{\alpha_{0}} f(t)\right|_{t=+0}=\left.I_{0+}^{\alpha_{0}-k} f(t)\right|_{t=+0}=0
$$

and therefore

$$
{ }^{c} D_{0+}^{\alpha_{i}} I_{0+}^{\alpha_{0}} f(t)=D_{0+}^{\alpha_{i}} I_{0+}^{\alpha_{0}} f(t)=D_{0+}^{\alpha_{i}} I_{0+}^{\alpha_{i}} I_{0+}^{\alpha_{0}-\alpha_{i}} f(t)=I_{0+}^{\alpha_{0}-\alpha_{i}} f(t) .
$$

Thus the second approximation is given as

$$
\begin{aligned}
y_{0}^{2}(t) & =\Phi_{1}(t)-I_{0+}^{\alpha_{0}} \sum_{i=1}^{m} a_{i}(t)^{c} D_{0+}^{\alpha_{i}} \Phi_{1}(t)+I_{0+}^{\alpha_{0}} \sum_{i=1}^{m} a_{i}(t) I_{0+}^{\alpha_{0}-\alpha_{i}} \sum_{i=h_{0}}^{m} a_{i}(t) \Phi_{1-\alpha_{i}}(t) \\
& =\Phi_{1}(t)+(-1)^{1} I_{0+}^{\alpha_{0}} \sum_{i=h_{0}}^{m} a_{i}(t) \Phi_{1-\alpha_{i}}(t)+(-1)^{2} I_{0+}^{\alpha_{0}} \sum_{i=1}^{m} a_{i}(t) I_{0+}^{\alpha_{0}-\alpha_{i}} \sum_{i=h_{0}}^{m} a_{i}(t) \Phi_{1-\alpha_{i}}(t) \\
& =\Phi_{1}(t)+\sum_{k=0}^{1}(-1)^{k+1} I_{0+}^{\alpha_{0}}\left[\sum_{i=1}^{m} a_{i}(t) I_{0+}^{\alpha_{0}-\alpha_{i}}\right]^{k} \sum_{i=h_{0}}^{m} a_{i}(t) \Phi_{1-\alpha_{i}}(t) .
\end{aligned}
$$

Since $h_{0}=m, \alpha_{m}=0$, we have $\Phi_{1-\alpha_{i}}(t)=\Phi_{1}(t) \in C[0, T]$ and therefore

$$
\begin{aligned}
D^{n_{0}} y_{0}^{2}(t) & =D^{n_{0}} \Phi_{1}(t)+\sum_{k=0}^{1}(-1)^{k+1} D^{n_{0}} I_{0+}^{\alpha_{0}}\left[\sum_{i=1}^{m} a_{i}(t) I_{0+}^{\alpha_{0}-\alpha_{i}}\right]^{k} \sum_{i=h_{0}}^{m} a_{i}(t) \Phi_{1-\alpha_{i}}(t) \\
& =\sum_{k=0}^{1}(-1)^{k+1} D I_{0+}^{\alpha_{0}-n_{0}+1}\left[\sum_{i=1}^{m} a_{i}(t) I_{0+}^{\alpha_{0}-\alpha_{i}}\right]^{k} \sum_{i=h_{0}}^{m} a_{i}(t) \Phi_{1-\alpha_{i}}(t) .
\end{aligned}
$$

Also we have

$$
I_{0+}^{\alpha_{0}-n_{0}+1}\left[\sum_{i=1}^{m} a_{i}(t) I_{0+}^{\alpha_{0}-\alpha_{i}}\right]^{k} \sum_{i=h_{0}}^{m} a_{i}(t) \Phi_{1-\alpha_{i}}(t) \in C_{\gamma}^{1}[0, T]
$$

Thus we have $y_{0}^{2}(t) \in C_{\gamma}^{n_{0}}[0, T]$.

Now under the assumption that the $l$ th approximation of $y_{0}(t)$ is provided by

$$
y_{0}^{l}(t)=\Phi_{1}(t)+\sum_{k=0}^{l-1}(-1)^{k+1} I_{0+}^{\alpha_{0}}\left[\sum_{i=1}^{m} a_{i}(t) I_{0+}^{\alpha_{0}-\alpha_{i}}\right]^{k} \sum_{i=h_{0}}^{m} a_{i}(t) \Phi_{1-\alpha_{i}}(t)
$$

and $y_{0}^{l}(t) \in C_{\gamma}^{n_{0}}[0, T]$, we find the $(l+1)$ th approximation of $y_{0}(t)$ :

$$
y_{0}^{l+1}(t)=\Phi_{1}(t)-I_{0+}^{\alpha_{0}} \sum_{i=1}^{m} a_{i}(t)^{c} D_{0+}^{\alpha_{i}} y_{0}^{l}(t)
$$




$$
\begin{aligned}
= & \Phi_{1}(t)-I_{0+}^{\alpha_{0}} \sum_{i=1}^{m} a_{i}(t)^{c} D_{0+}^{\alpha_{i}}\left\{\Phi_{1}(t)\right. \\
& \left.+\sum_{k=0}^{l-1}(-1)^{k+1} I_{0+}^{\alpha_{0}}\left[\sum_{i=1}^{m} a_{i}(t) I_{0+}^{\alpha_{0}-\alpha_{i}}\right]^{k} \sum_{i=h_{0}}^{m} a_{i}(t) \Phi_{1-\alpha_{i}}(t)\right\} \\
= & \Phi_{1}(t)-I_{0+}^{\alpha_{0}} \sum_{i=h_{0}}^{m} a_{i}(t) \Phi_{1-\alpha_{i}}(t) \\
& -I_{0+}^{\alpha_{0}} \sum_{i=1}^{m} a_{i}(t) \sum_{k=0}^{l-1}(-1)^{k+1} I_{0+}^{\alpha_{0}-\alpha_{i}}\left[\sum_{i=1}^{m} a_{i}(t) I_{0+}^{\alpha_{0}-\alpha_{i}}\right]^{k} \sum_{i=h_{0}}^{m} a_{i}(t) \Phi_{1-\alpha_{i}}(t) \\
= & \Phi_{1}(t)+\sum_{k=0}^{l}(-1)^{k+1} I_{0+}^{\alpha_{0}}\left[\sum_{i=1}^{m} a_{i}(t) I_{0+}^{\alpha_{0}-\alpha_{i}}\right]^{k} \sum_{i=h_{0}}^{m} a_{i}(t) \Phi_{1-\alpha_{i}}(t) .
\end{aligned}
$$

Thus the $(l+1)$ th approximation of $y_{0}(t)$ is provided by

$$
y_{0}^{l+1}(t)=\Phi_{1}(t)+\sum_{k=0}^{l}(-1)^{k+1} I_{0+}^{\alpha_{0}}\left[\sum_{i=1}^{m} a_{i}(t) I_{0+}^{\alpha_{0}-\alpha_{i}}\right]^{k} \sum_{i=h_{0}}^{m} a_{i}(t) \Phi_{1-\alpha_{i}}(t) .
$$

Similar to the above, we get $y_{0}^{l+1}(t) \in C_{\gamma}^{n_{0}}[0, T]$.

By induction, we proved that, for any $n=0,1, \ldots$, the $n$th approximation of $y_{0}(t)$ is provided by

$$
y_{0}^{n}(t)=\Phi_{1}(t)+\sum_{k=0}^{n-1}(-1)^{k+1} I_{0+}^{\alpha_{0}}\left[\sum_{i=1}^{m} a_{i}(t) I_{0+}^{\alpha_{0}-\alpha_{i}}\right]^{k} \sum_{i=h_{0}}^{m} a_{i}(t) \Phi_{1-\alpha_{i}}(t)
$$

and $y_{0}^{n}(t) \in C_{\gamma}^{n_{0}}[0, T]$. The sequence $\left\{y_{0}^{n}(t)\right\}$ converges in $C_{\gamma}^{n_{0}}[0, T]$ and we get as the first element $y_{0}(t)$ of the canonical system

$$
\begin{aligned}
y_{0}(t) & =\lim _{n \rightarrow \infty} y_{0}^{n}(t) \\
& =\Phi_{1}(t)+\sum_{k=0}^{\infty}(-1)^{k+1} I_{0+}^{\alpha_{0}}\left[\sum_{i=1}^{m} a_{i}(t) I_{0+}^{\alpha_{0}-\alpha_{i}}\right]^{k} \sum_{i=h_{0}}^{m} a_{i}(t) \Phi_{1-\alpha_{i}}(t) \in C_{\gamma}^{n_{0}}[0, T] .
\end{aligned}
$$

Now for any $j\left(j=1, \ldots, n_{0}-1\right)$, we find the $j$ th element $y_{j}(t)$ of the canonical system. From (11) and (12), the first approximation of $y_{j}(t)$ is given by

$$
y_{j}^{1}(t)=\Phi_{j+1}(t)-I_{0+}^{\alpha_{0}} \sum_{i=1}^{m} a_{i}(t)^{c} D_{0+}^{\alpha_{i}} y_{j}^{0}(t)=\Phi_{j+1}(t)-I_{0+}^{\alpha_{0}} \sum_{i=1}^{m} a_{i}(t)^{c} D_{0+}^{\alpha_{i}} \Phi_{j+1}(t) .
$$

Also ${ }^{c} D_{0+}^{\alpha_{i}} \Phi_{j+1}(t)$ is represented as

$$
{ }^{c} D_{0+}^{\alpha_{i}} \Phi_{j+1}(t)=D_{0+}^{\alpha_{i}}\left[\Phi_{j+1}(t)-\sum_{k=0}^{n_{i}-1} D^{k} \Phi_{j+1}(0) \Phi_{k+1}(t)\right], \quad i=1, \ldots, m
$$


If $i \geq h_{j}, 0 \leq \alpha_{i} \leq j$ and $n_{i} \leq j$. Thus $D^{k} \Phi_{j+1}(0)=0, k=0, \ldots, n_{0}-1$. That is, we get

$$
{ }^{c} D_{0+}^{\alpha_{i}} \Phi_{j+1}(t)=D_{0+}^{\alpha_{i}} \Phi_{j+1}(t), \quad i=h_{j}, \ldots, m
$$

If $i<h_{j}$, then $n_{i}>j$ and so

$$
\sum_{k=0}^{n_{i}-1} D^{k} \Phi_{j+1}(0) \Phi_{k+1}(t)=\Phi_{j+1}(t)
$$

and thus ${ }^{c} D_{0+}^{\alpha_{i}} \Phi_{j+1}(t)=0, i<h_{j}$. That is, we get

$$
{ }^{c} D_{0+}^{\alpha_{i}} \Phi_{j+1}(t)= \begin{cases}D_{0+}^{\alpha_{i}} \Phi_{j+1}(t), & h_{j} \leq i \leq m, \\ 0, & 1 \leq i<h_{j} .\end{cases}
$$

Therefore the first approximation of $y_{j}(t)$ is provided by

$$
\begin{aligned}
y_{j}^{1}(t) & =\Phi_{j+1}(t)-I_{0+}^{\alpha_{0}} \sum_{i=1}^{m} a_{i}(t)^{c} D_{0+}^{\alpha_{i}} \Phi_{j+1}(t)=\Phi_{j+1}(t)-I_{0+}^{\alpha_{0}} \sum_{i=h_{j}}^{m} a_{i}(t) D_{0+}^{\alpha_{i}} \Phi_{j+1}(t) \\
& =\Phi_{j+1}(t)-I_{0+}^{\alpha_{0}} \sum_{i=h_{j}}^{m} a_{i}(t) \Phi_{j+1-\alpha_{i}}(t)
\end{aligned}
$$

where

$$
\Phi_{j+1-\alpha_{i}}(t):=D_{0+}^{\alpha_{i}} \Phi_{j+1}(t) .
$$

We have $y_{j}^{1}(t) \in C_{\gamma}^{n_{0}}[0, T]$.

The second approximation of $y_{j}(t)$ is given by

$$
\begin{aligned}
y_{j}^{2}(t) & =\Phi_{j+1}(t)-I_{0+}^{\alpha_{0}} \sum_{i=1}^{m} a_{i}(t)^{c} D_{0+}^{\alpha_{i}} y_{j}^{1}(t) \\
& =\Phi_{j+1}(t)-I_{0+}^{\alpha_{0}} \sum_{i=1}^{m} a_{i}(t)^{c} D_{0+}^{\alpha_{i}}\left[\Phi_{j+1}(t)-I_{0+}^{\alpha_{0}} \sum_{i=h_{j}}^{m} a_{i}(t) \Phi_{j+1-\alpha_{i}}(t)\right] \\
& =\Phi_{j+1}(t)-I_{0+}^{\alpha_{0}} \sum_{i=1}^{m} a_{i}(t)^{c} D_{0+}^{\alpha_{i}} \Phi_{j+1}(t)+I_{0+}^{\alpha_{0}} \sum_{i=1}^{m} a_{i}(t)^{c} D_{0+}^{\alpha_{i}} I_{0+}^{\alpha_{0}} \sum_{i=h_{j}}^{m} a_{i}(t) \Phi_{j+1-\alpha_{i}}(t) .
\end{aligned}
$$

Here since ${ }^{c} D_{0+}^{\alpha_{i}} I_{0+}^{\alpha_{0}} \sum_{i=h_{j}}^{m} a_{i}(t) \Phi_{j+1-\alpha_{i}}(t)=I_{0+}^{\alpha_{0}-\alpha_{i}} \sum_{i=h_{j}}^{m} a_{i}(t) \Phi_{j+1-\alpha_{i}}(t)$, we get

$$
y_{j}^{2}(t)=\Phi_{j+1}(t)+\sum_{k=0}^{1}(-1)^{k+1} I_{0+}^{\alpha_{0}}\left[\sum_{i=1}^{m} a_{i}(t) I_{0+}^{\alpha_{0}-\alpha_{i}}\right]^{k} \sum_{i=h_{j}}^{m} a_{i}(t) \Phi_{j+1-\alpha_{i}}(t) .
$$

By induction, the $n$th approximation of $y_{j}(t)$ is given by

$$
y_{j}^{n}(t)=\Phi_{j+1}(t)+\sum_{k=0}^{n-1}(-1)^{k+1} I_{0+}^{\alpha_{0}}\left[\sum_{i=1}^{m} a_{i}(t) I_{0+}^{\alpha_{0}-\alpha_{i}}\right]^{k} \sum_{i=h_{j}}^{m} a_{i}(t) \Phi_{j+1-\alpha_{i}}(t) .
$$


Therefore

$$
y_{j}(t)=\lim _{n \rightarrow \infty} y_{j}^{n}(t)=\Phi_{j+1}(t)+\sum_{k=0}^{\infty}(-1)^{k+1} I_{0+}^{\alpha_{0}}\left[\sum_{i=1}^{m} a_{i}(t) I_{0+}^{\alpha_{0}-\alpha_{i}}\right]^{k} \sum_{i=h_{j}}^{m} a_{i}(t) \Phi_{j+1-\alpha_{i}}(t) .
$$

Lemma 3.3 Let us assume that $n_{0}>n_{1}, a_{i} \in C[0, T](i=1, \ldots, m)$ and $\boldsymbol{H}_{0} \neq \varnothing$. Then there exists the unique canonical fundamental system

$$
y_{j}(t) \in C^{\alpha_{0}, n_{0}-1}[0, T], \quad j=0,1, \ldots, n_{0}-1,
$$

of solutions for Eq. (3) and it is written by

$$
\begin{aligned}
y_{j}(t) & =\Phi_{j+1}(t)+\sum_{k=0}^{\infty}(-1)^{k+1} I_{0+}^{\alpha_{0}}\left[\sum_{i=1}^{m} a_{i}(t) I_{0+}^{\alpha_{0}-\alpha_{i}}\right]^{k} \sum_{i=h_{j}}^{m} a_{i}(t) \Phi_{j+1-\alpha_{i}}(t), \\
j & =0,1, \ldots, n_{1}-1, \\
y_{j}(t) & =\Phi_{j+1}(t)+\sum_{k=0}^{\infty}(-1)^{k+1} I_{0+}^{\alpha_{0}}\left[\sum_{i=1}^{m} a_{i}(t) I_{0+}^{\alpha_{0}-\alpha_{i}}\right]^{k} \sum_{i=1}^{m} a_{i}(t) \Phi_{j+1-\alpha_{i}}(t), \\
j & =n_{1}, n_{1}+1, \ldots, n_{0}-1 .
\end{aligned}
$$

Proof Let find the canonical system as the limit of the approximation sequence (11) and (12). Fixing $j=0,1, \ldots, n_{0}-1$, we find the $j$ th element $y_{j}(t)$ of the canonical system.

$$
\begin{aligned}
& y_{j}^{0}(t)=\Phi_{j+1}(t), \\
& y_{j}^{1}(t)=\Phi_{j+1}(t)-I_{0+}^{\alpha_{0}} \sum_{i=1}^{m} a_{i}(t)^{c} D_{0}^{\alpha_{i}} y_{j}^{0}(t)=\Phi_{j+1}(t)-I_{0+}^{\alpha_{0}} \sum_{i=1}^{m} a_{i}(t)^{c} D_{0}^{\alpha_{i}} \Phi_{j+1}(t) .
\end{aligned}
$$

Let us note that, for $k=0,1, \ldots, n_{i}-1$,

$$
D^{k} \Phi_{j+1}(0)= \begin{cases}1, & k=j \\ 0, & k \neq j\end{cases}
$$

Since $n_{0}>n_{1} \geq n_{i}$, for $j=0,1, \ldots, n_{1}-1$, we get

$$
{ }^{c} D_{0+}^{\alpha_{i}} \Phi_{j+1}(t)= \begin{cases}D_{0+}^{\alpha_{i}} \Phi_{j+1}(t), & h_{j} \leq i \leq m \\ 0, & 1 \leq i<h_{j}\end{cases}
$$

If $j=n_{1}, \ldots, n_{0}-1$, then $k<j$ and thus we get

$$
{ }^{c} D_{0+}^{\alpha_{i}} \Phi_{j+1}(t)=D_{0+}^{\alpha_{i}} \Phi_{j+1}(t), \quad i=1, \ldots, m .
$$


Therefore the first approximation of $y_{j}(t)$ is given by

$$
\begin{aligned}
& y_{j}^{1}(t)=\Phi_{j+1}(t)-I_{0+}^{\alpha_{0}} \sum_{i=h_{j}}^{m} a_{i}(t) D_{0+}^{\alpha_{i}} \Phi_{j+1}(t)=\Phi_{j+1}(t)-I_{0+}^{\alpha_{0}} \sum_{i=h_{j}}^{m} a_{i}(t) \Phi_{j+1-\alpha_{i}}(t), \\
& j=0,1, \ldots, n_{1}-1, \\
& y_{j}^{1}(t)=\Phi_{j+1}(t)-I_{0+}^{\alpha_{0}} \sum_{i=1}^{m} a_{i}(t) D_{0+}^{\alpha_{i}} \Phi_{j+1}(t)=\Phi_{j+1}(t)-I_{0+}^{\alpha_{0}} \sum_{i=1}^{m} a_{i}(t) \Phi_{j+1-\alpha_{i}}(t), \\
& j=n_{1}, \ldots, n_{0}-1 .
\end{aligned}
$$

We obtain $y_{j}^{1}(t) \in C^{\alpha_{0}, n_{0}-1}[0, T], j=0,1, \ldots, n_{0}-1$.

For $j=0,1, \ldots, n_{1}-1$, the second approximation is given as

$$
\begin{aligned}
y_{j}^{2}(t) & =\Phi_{j+1}(t)-I_{0+}^{\alpha_{0}} \sum_{i=1}^{m} a_{i}(t)^{c} D_{0+}^{\alpha_{i}} y_{j}^{1}(t) \\
& =\Phi_{j+1}(t)-I_{0+}^{\alpha_{0}} \sum_{i=1}^{m} a_{i}(t)^{c} D_{0+}^{\alpha_{i}} \Phi_{j+1}(t)+I_{0+}^{\alpha_{0}} \sum_{i=1}^{m} a_{i}(t)^{c} D_{0+}^{\alpha_{i}} I_{0+}^{\alpha_{0}} \sum_{i=h_{j}}^{m} a_{i}(t) \Phi_{j+1-\alpha_{i}}(t) .
\end{aligned}
$$

By Eq. (18) and the fact that ${ }^{c} D_{0+}^{\alpha_{i}} I_{0+}^{\alpha_{0}} \sum_{i=h_{j}}^{m} a_{i}(t) \Phi_{j+1-\alpha_{i}}(t)=I_{0+}^{\alpha_{0}-\alpha_{i}} \sum_{i=h_{j}}^{m} a_{i}(t) \Phi_{j+1-\alpha_{i}}(t)$, we can rewrite (20) as

$$
\begin{aligned}
y_{j}^{2}(t) & =\Phi_{j+1}(t)-I_{0+}^{\alpha_{0}} \sum_{i=h_{j}}^{m} a_{i}(t) D_{0+}^{\alpha_{i}} \Phi_{j+1}(t)+I_{0+}^{\alpha_{0}} \sum_{i=1}^{m} a_{i}(t) I_{0+}^{\alpha_{0}-\alpha_{i}} \sum_{i=h_{j}}^{m} a_{i}(t) \Phi_{j+1-\alpha_{i}}(t) \\
& =\Phi_{j+1}(t)+\sum_{k=0}^{1}(-1)^{k+1} I_{0+}^{\alpha_{0}}\left[\sum_{i=1}^{m} a_{i}(t) I_{0+}^{\alpha_{0}-\alpha_{i}}\right]^{k} \sum_{i=h_{j}}^{m} a_{i}(t) \Phi_{j+1-\alpha_{i}}(t) .
\end{aligned}
$$

If $j=n_{1}, n_{1}+1, \ldots, n_{0}-1$, then the second approximation is given as

$$
\begin{aligned}
y_{j}^{2}(t) & =\Phi_{j+1}(t)-I_{0+}^{\alpha_{0}} \sum_{i=1}^{m} a_{i}(t)^{c} D_{0+}^{\alpha_{i}} y_{j}^{1}(t) \\
& =\Phi_{j+1}(t)+\sum_{k=0}^{1}(-1)^{k+1} I_{0+}^{\alpha_{0}}\left[\sum_{i=1}^{m} a_{i}(t) I_{0+}^{\alpha_{0}-\alpha_{i}}\right]^{k} \sum_{i=1}^{m} a_{i}(t) \Phi_{j+1-\alpha_{i}}(t) .
\end{aligned}
$$

Thus we have the representation of the second approximation of $y_{j}(t)$ as

$$
\begin{aligned}
& y_{j}^{2}(t)=\Phi_{j+1}(t)+\sum_{k=0}^{1}(-1)^{k+1} I_{0+}^{\alpha_{0}}\left[\sum_{i=1}^{m} a_{i}(t) I_{0+}^{\alpha_{0}-\alpha_{i}}\right]^{k} \sum_{i=h_{j}}^{m} a_{i}(t) \Phi_{j+1-\alpha_{i}}(t), \\
& j=0,1, \ldots, n_{1}-1, \\
& y_{j}^{2}(t)=\Phi_{j+1}(t)+\sum_{k=0}^{1}(-1)^{k+1} I_{0+}^{\alpha_{0}}\left[\sum_{i=1}^{m} a_{i}(t) I_{0+}^{\alpha_{0}-\alpha_{i}}\right]^{k} \sum_{i=1}^{m} a_{i}(t) \Phi_{j+1-\alpha_{i}}(t), \\
& j=n_{1}, n_{1}+1, \ldots, n_{0}-1 .
\end{aligned}
$$


For $n=3,4, \ldots$, we can find $y_{j}^{n}(t)$ by induction and, by letting $n \rightarrow \infty$, we get the representation (16) and (17) of the canonical fundamental system.

Lemma 3.4 Let $0<\gamma=n_{0}-\alpha_{0}<1, n_{0}=n_{1}, a_{i} \in C_{\gamma}^{1}[0, T]$ and $D_{0+}^{n_{0}-\alpha_{0}} a_{i} \in C[0, T], i=$ $1, \ldots, m$. Let assume that Eq. (3) is of type 2. That is, let assume that $n_{0} \geq 2$ and there exists a $j_{0} \in\left\{0,1, \ldots, n_{0}-2\right\}$ such that $\boldsymbol{H}_{j_{0}}=\varnothing$ and $\boldsymbol{H}_{j_{0}+1} \neq \varnothing$. Then there exists the unique canonical fundamental system $y_{j}(t) \in C_{\gamma}^{n_{0}}[0, T], j=0,1, \ldots, n_{0}-1$ of solutions for Eq. (3) and it is written by

$$
\begin{aligned}
y_{j}(t) & =\Phi_{j+1}(t), \quad j=0,1, \ldots, j_{0} \\
y_{j}(t) & =\Phi_{j+1}(t)+\sum_{k=0}^{\infty}(-1)^{k+1} I_{0+}^{\alpha_{0}}\left[\sum_{i=1}^{m} a_{i}(t) I_{0+}^{\alpha_{0}-\alpha_{i}}\right]^{k} \sum_{i=h_{j}}^{m} a_{i}(t) \Phi_{j+1-\alpha_{i}}(t), \\
j & =j_{0}+1, \ldots, n_{0}-1 .
\end{aligned}
$$

Proof Similarly, we can see that the first approximation of $y_{j}(t)$ is written

$$
\begin{aligned}
& y_{j}^{1}(t)=\Phi_{j+1}(t), \quad j=0,1, \ldots, j_{0}, \\
& y_{j}^{1}(t)=\Phi_{j+1}(t)-I_{0+}^{\alpha_{0}} \sum_{i=h_{j}}^{m} a_{i}(t) \Phi_{j+1-\alpha_{i}}(t), \quad j=j_{0}+1, \ldots, n_{0}-1 .
\end{aligned}
$$

We get $y_{j}^{1}(t) \in C_{\gamma}^{n_{0}}[0, T], j=j_{0}+1, \ldots, n_{0}-1$.

Also the second approximation of $y_{j}(t)$ is given as

$$
\begin{aligned}
& y_{j}^{2}(t)=\Phi_{j+1}(t), \quad j=0,1, \ldots, j_{0} \\
& y_{j}^{2}(t)=\Phi_{j+1}(t)+\sum_{k=0}^{1}(-1)^{k+1} I_{0+}^{\alpha_{0}}\left[\sum_{i=1}^{m} a_{i}(t) I_{0+}^{\alpha_{0}-\alpha_{i}}\right]^{k} \sum_{i=h_{j}}^{m} a_{i}(t) \Phi_{j+1-\alpha_{i}}(t), \\
& j=j_{0}+1, \ldots, n_{0}-1 .
\end{aligned}
$$

By induction, we get the $n$th approximation of $y_{j}(t)$,

$$
\begin{aligned}
& y_{j}^{n}(t)=\Phi_{j+1}(t), \quad j=0,1, \ldots, j_{0} \\
& y_{j}^{n}(t)=\Phi_{j+1}(t)+\sum_{k=0}^{n-1}(-1)^{k+1} I_{0+}^{\alpha_{0}}\left[\sum_{i=1}^{m} a_{i}(t) I_{0+}^{\alpha_{0}-\alpha_{i}}\right]^{k} \sum_{i=h_{j}}^{m} a_{i}(t) \Phi_{j+1-\alpha_{i}}(t), \\
& j=j_{0}+1, \ldots, n_{0}-1 .
\end{aligned}
$$

That is, the canonical fundamental system of solutions for Eq. (3) is given by (21) and (22) and we get $y_{j}(t) \in C_{\gamma}^{n_{0}}[0, T], j=0,1, \ldots, n_{0}-1$.

Lemma 3.5 Lets assume that $n_{0}>n_{1}$ and $a_{i} \in[0, T], i=1, \ldots, m$. Lets assume that Eq. (3) is the type 2. That is, assume that $n_{0} \geq 2$ and there exists $a j_{0} \in\left\{0,1, \ldots, n_{0}-2\right\}$ such that 
$\boldsymbol{H}_{j_{0}}=\varnothing$ and $\boldsymbol{H}_{j_{0}+1} \neq \varnothing$. Then there exists the unique canonical fundamental system $y_{j}(t) \in$ $C^{\alpha_{0}, n_{0}-1}[0, T], j=0,1, \ldots, n_{0}-1$ of solution for Eq. (3) and it is written by

$$
\begin{aligned}
y_{j}(t) & =\Phi_{j+1}(t), \quad j=0,1, \ldots, j_{0} \\
y_{j}(t) & =\Phi_{j+1}(t)+\sum_{k=0}^{\infty}(-1)^{k+1} I_{0+}^{\alpha_{0}}\left[\sum_{i=1}^{m} a_{i}(t) I_{0+}^{\alpha_{0}-\alpha_{i}}\right]^{k} \sum_{i=h_{j}}^{m} a_{i}(t) \Phi_{j+1-\alpha_{i}}(t), \\
j & =j_{0}+1, \ldots, n_{1}-1, \\
y_{j}(t) & =\Phi_{j+1}(t)+\sum_{k=0}^{\infty}(-1)^{k+1} I_{0+}^{\alpha_{0}}\left[\sum_{i=1}^{m} a_{i}(t) I_{0+}^{\alpha_{0}-\alpha_{i}}\right]^{k} \sum_{i=1}^{m} a_{i}(t) \Phi_{j+1-\alpha_{i}}(t), \\
j & =n_{1}, n_{1}+1, \ldots, n_{0}-1 .
\end{aligned}
$$

Proof Similarly, we can see the first approximation of $y_{j}(t)$ is given by

$$
\begin{aligned}
& y_{j}^{1}(t)=\Phi_{j+1}(t), \quad j=0,1, \ldots, j_{0}, \\
& y_{j}^{1}(t)=\Phi_{j+1}(t)-I_{0+}^{\alpha_{0}} \sum_{i=h_{j}}^{m} a_{i}(t) \Phi_{j+1-\alpha_{i}}(t), \quad j=j_{0}+1, \ldots, n_{1}-1, \\
& y_{j}^{1}(t)=\Phi_{j+1}(t)-I_{0+}^{\alpha_{0}} \sum_{i=1}^{m} a_{i}(t) \Phi_{j+1-\alpha_{i}}(t), \quad j=n_{1}, \ldots, n_{0}-1 .
\end{aligned}
$$

Also we get the second approximation of $y_{j}^{1}(t)$,

$$
\begin{aligned}
& y_{j}^{2}(t)=\Phi_{j+1}(t), \quad j=0,1, \ldots, j_{0}, \\
& y_{j}^{2}(t)=\Phi_{j+1}(t)+\sum_{k=0}^{1}(-1)^{k+1} I_{0+}^{\alpha_{0}}\left[\sum_{i=1}^{m} a_{i}(t) I_{0+}^{\alpha_{0}-\alpha_{i}}\right]^{k} \sum_{i=h_{j}}^{m} a_{i}(t) \Phi_{j+1-\alpha_{i}}(t), \\
& j=j_{0}+1, \ldots, n_{1}-1, \\
& y_{j}^{2}(t)=\Phi_{j+1}(t)+\sum_{k=0}^{1}(-1)^{k+1} I_{0+}^{\alpha_{0}}\left[\sum_{i=1}^{m} a_{i}(t) I_{0+}^{\alpha_{0}-\alpha_{i}}\right]^{k} \sum_{i=1}^{m} a_{i}(t) \Phi_{j+1-\alpha_{i}}(t), \\
& j=n_{1}, n_{1}+1, \ldots, n_{0}-1 .
\end{aligned}
$$

In a similar way to the above proof, we can get the $n$th approximation of $y_{j}(t)$ and then, taking $n \rightarrow \infty$, we can get the canonical fundamental system (23) and (24) and (25) and $y_{j}(t) \in C^{\alpha_{0}, n_{0}-1}[0, T], j=0,1, \ldots, n_{0}-1$.

Lemma 3.6 Let $0<\gamma=n_{0}-\alpha_{0}<1$ and assume that $a_{i} \in C_{\gamma}^{1}[0, T], i=1, \ldots, m$. Let us assume that Eq. (3) is of type 3. That is, let assume that $\boldsymbol{H}_{n_{0}-1}=\varnothing$. Then Eq. (3) has the unique canonical fundamental system $\left\{y_{j}(t): j=0,1, \ldots, n_{0}-1\right\}$ of solutions in $C_{\gamma}^{n_{0}}[0, T]$ and it is represented as

$$
y_{j}(t)=\Phi_{j+1}(t), \quad j=0,1, \ldots, n_{0}-1
$$


Proof Let $y_{j}^{0}(t)=\Phi_{j+1}(t), j=0,1, \ldots, n_{0}-1$ and calculate the first approximation of $y_{j}(t)$. Then we have

$$
y_{j}^{1}(t)=\Phi_{j+1}(t)-I_{0+}^{\alpha_{0}} \sum_{i=1}^{m} a_{i}(t)^{c} D_{0+}^{\alpha_{i}} \Phi_{j+1}(t)
$$

From the assumption of $H_{n_{0}-1}=\left\{i: 0 \leq \alpha_{i} \leq n_{0}-1, i=1, \ldots, m\right\}=\varnothing$, it is evident that $n_{0}-1<\alpha_{i} \leq n_{0}$ for all $i=1, \ldots, m$ and thus

$$
{ }^{c} D_{0+}^{\alpha_{i}} \Phi_{j+1}(t)=D_{0+}^{\alpha_{i}}\left[\Phi_{j+1}(t)-\sum_{k=0}^{n_{0}-1} D^{k} \Phi_{j+1}(0) \Phi_{k+1}(t)\right]=0, \quad j=1, \ldots, n_{0}-1
$$

and $y_{j}^{1}(t)=\Phi_{j+1}(t), j=1, \ldots, n_{0}-1$. Similarly, we can easily find

$$
y_{j}^{n}(t)=\Phi_{j+1}(t), \quad j=0, \ldots, n_{0}-1,
$$

and thus we can get

$$
y_{j}(t)=\Phi_{j+1}(t) \in C_{\gamma}^{n_{0}}[0, T], \quad j=0, \ldots, n_{0}-1
$$

Theorem 3.1 Let $g(t), a_{i}(t) \in C[0, T], i=1, \ldots, m$. Then there exists the unique solution $y(t) \in C^{\alpha_{0}, n_{0}-1}[0, T]$ of the initial value problem (1) and (4) and it is represented by

$$
y(t)=\sum_{k=0}^{\infty}(-1)^{k} I_{0+}^{\alpha_{0}}\left[\sum_{i=1}^{m} a_{i}(t) I_{0+}^{\alpha_{0}-\alpha_{i}}\right]^{k} g(t) .
$$

Proof From the assumptions and Lemma 3.1 follow the existence and uniqueness of the solution of Eqs. (1) and (4). Now we have to find this solution.

From Lemma 3.1, the first approximation solution is given by

$$
y^{1}(t)=I_{0+}^{\alpha_{0}} g(t)-I_{0+}^{\alpha_{0}} \sum_{i=1}^{m} a_{i}(t)^{c} D_{0+}^{\alpha_{i}} I_{0+}^{\alpha_{0}} g(t)
$$

With a view on the definition of the Caputo fractional derivative and the integrability of $I_{0+}^{\alpha_{0}-\alpha_{i}} g(t)$, we have

$$
\begin{aligned}
{ }^{c} D_{0+}^{\alpha_{i}} I_{0+}^{\alpha_{0}} g(t) & =D_{0+}^{\alpha_{i}}\left[I_{0+}^{\alpha_{0}} g(t)-\sum_{j=1}^{n_{i}-1}\left(D^{j} I_{0+}^{\alpha_{0}} g\right)(0) \Phi_{j+1}(t)\right]=D_{0+}^{\alpha_{i}} I_{0+}^{\alpha_{0}} g(t) \\
& =D_{0+}^{\alpha_{i}} I_{0+}^{\alpha_{i}} I_{0+}^{\alpha_{0}-\alpha_{i}} g(t)=I_{0+}^{\alpha_{0}-\alpha_{i}} g(t) .
\end{aligned}
$$

So, the first approximation solution can be rewritten

$$
\begin{aligned}
y^{1}(t) & =I_{0+}^{\alpha_{0}} g(t)-I_{0+}^{\alpha_{0}} \sum_{i=1}^{m} a_{i}(t) I_{0+}^{\alpha_{0}-\alpha_{i}} g(t)=I_{0+}^{\alpha_{0}} g(t)-I_{0+}^{\alpha_{0}}\left[\sum_{i=1}^{m} a_{i}(t) I_{0+}^{\alpha_{0}-\alpha_{i}}\right] g(t) \\
& =\sum_{k=0}^{1}(-1)^{k} I_{0_{+}}^{\alpha_{0}}\left[\sum_{i=1}^{m} a_{i}(t) I_{0+}^{\alpha_{0}-\alpha_{i}}\right]^{k} g(t),
\end{aligned}
$$




$$
D^{n_{0}-1} y^{1}(t)=I_{0+}^{\alpha_{0}-n_{0}+1} g(t)-I_{0+}^{\alpha_{0}-n_{0}+1}\left[\sum_{i=1}^{m} a_{i}(t) I_{0+}^{\alpha_{0}-\alpha_{i}}\right] g(t),
$$

where $\alpha_{0}-n_{0}+1>0, g(t) \in C[0, T]$ and $\left[\sum_{i=1}^{m} a_{i}(t) I_{0+}^{\alpha_{0}-\alpha_{i}}\right] g(t) \in C[0, T]$.

Hence the Lemma 3.1 yields $D^{n_{0}-1} y^{1}(t) \in C[0, T]$, that is, $y^{1}(t) \in C^{n_{0}-1}[0, T]$.

Moreover, we have

$$
\begin{aligned}
{ }^{c} D_{0+}^{\alpha_{0}} y^{1}(t)= & { }^{c} D_{0+}^{\alpha_{0}}\left[I_{0+}^{\alpha_{0}} g(t)-I_{0+}^{\alpha_{0}}\left[\sum_{i=1}^{m} a_{i}(t) I_{0+}^{\alpha_{0}-\alpha_{i}}\right] g(t)\right] \\
= & D_{0+}^{\alpha_{0}}\left[I_{0+}^{\alpha_{0}} g(t)-I_{0+}^{\alpha_{0}}\left[\sum_{i=1}^{m} a_{i}(t) I_{0+}^{\alpha_{0}-\alpha_{i}}\right] g(t)\right. \\
& \left.-\left.\sum_{j=1}^{n_{0}-1} D^{j}\left[I_{0+}^{\alpha_{0}} g(t)-I_{0+}^{\alpha_{0}}\left[\sum_{i=1}^{m} a_{i}(t) I_{0+}^{\alpha_{0}-\alpha_{i}}\right] g(t)\right]\right|_{t=0} \Phi_{j+1}(t)\right] \\
= & g(t)-\left[\sum_{i=1}^{m} a_{i}(t) I_{0+}^{\alpha_{0}-\alpha_{i}}\right] g(t) \in C[0, T],
\end{aligned}
$$

from which $y^{1}(t) \in C^{\alpha_{0}, n_{0}-1}[0, T]$.

Next, we find the second approximation solution, i.e., $l=2$ in the expression of Eq. (6):

$$
\begin{aligned}
y^{2}(t) & =I_{0+}^{\alpha_{0}} g(t)-I_{0+}^{\alpha_{0}} \sum_{i=1}^{m} a_{i}(t)^{c} D_{0+}^{\alpha_{i}} y^{1}(t) \\
& =I_{0+}^{\alpha_{0}} g(t)-I_{0+}^{\alpha_{0}} \sum_{i=1}^{m} a_{i}(t)^{c} D_{0+}^{\alpha_{i}}\left[I_{0+}^{\alpha_{0}} g(t)-I_{0+}^{\alpha_{0}}\left[\sum_{i=1}^{m} a_{i}(t) I_{0+}^{\alpha_{0}-\alpha_{i}}\right] g(t)\right] \\
& =I_{0+}^{\alpha_{0}} g(t)-I_{0+}^{\alpha_{0}} \sum_{i=1}^{m} a_{i}(t) I_{0+}^{\alpha_{0}-\alpha_{i}} g(t)+I_{0+}^{\alpha_{0}} \sum_{i=1}^{m} a_{i}(t) I_{0+}^{\alpha_{0}-\alpha_{i}}\left[\sum_{i=1}^{m} a_{i}(t) I_{0+}^{\alpha_{0}-\alpha_{i}}\right] g(t) \\
& =I_{0+}^{\alpha_{0}} g(t)-I_{0+}^{\alpha_{0}} \sum_{i=1}^{m} a_{i}(t) I_{0+}^{\alpha_{0}-\alpha_{i}} g(t)+I_{0+}^{\alpha_{0}}\left[\sum_{i=1}^{m} a_{i}(t) I_{0+}^{\alpha_{0}-\alpha_{i}}\right]^{2} g(t) \\
& =\sum_{k=0}^{2}(-1)^{k} I_{0+}^{\alpha_{0}}\left[\sum_{i=1}^{m} a_{i}(t) I_{0+}^{\alpha_{0}-\alpha_{i}}\right]^{k} g(t) .
\end{aligned}
$$

Thus, we get the second approximation solution

$$
y^{2}(t)=\sum_{k=0}^{2}(-1)^{k} I_{0+}^{\alpha_{0}}\left[\sum_{i=1}^{m} a_{i}(t) I_{0+}^{\alpha_{0}-\alpha_{i}}\right]^{k} g(t) .
$$

Now, we inductively find the $(l+1)$ th approximation solution when the $l$ th approximation solution for any $l \in \mathbf{N}$ is provided by

$$
y^{l}(t)=\sum_{k=0}^{l}(-1)^{k} I_{0+}^{\alpha_{0}}\left[\sum_{i=1}^{m} a_{i}(t) I_{0+}^{\alpha_{0}-\alpha_{i}}\right]^{k} g(t) .
$$


Then we have

$$
\begin{aligned}
y^{l+1}(t) & =I_{0+}^{\alpha_{0}} g(t)-I_{0+}^{\alpha_{0}} \sum_{i=1}^{m} a_{i}(t)^{c} D_{0+}^{\alpha_{i}}\left[\sum_{k=0}^{l}(-1)^{k} I_{0+}^{\alpha_{0}}\left[\sum_{i=1}^{m} a_{i}(t) I_{0+}^{\alpha_{0}-\alpha_{i}}\right]^{k} g(t)\right] \\
& =I_{0+}^{\alpha_{0}} g(t)-I_{0+}^{\alpha_{0}}\left[\sum_{k=0}^{l}(-1)^{k} \sum_{i=1}^{m} a_{i}(t)^{c} D_{0+}^{\alpha_{i}} I_{0+}^{\alpha_{0}}\left[\sum_{i=1}^{m} a_{i}(t) I_{0+}^{\alpha_{0}-\alpha_{i}}\right]^{k} g(t)\right] \\
& =I_{0+}^{\alpha_{0}} g(t)-I_{0+}^{\alpha_{0}}\left[\sum_{k=0}^{l}(-1)^{k} \sum_{i=1}^{m} a_{i}(t) I_{0+}^{\alpha_{0}-\alpha}\left[\sum_{i=1}^{m} a_{i}(t) I_{0+}^{\alpha_{0}-\alpha_{i}}\right]^{k} g(t)\right] \\
& =\sum_{k=0}^{l+1}(-1)^{k} I_{0+}^{\alpha_{0}}\left[\sum_{i=1}^{m} a_{i}(t) I_{0+}^{\alpha_{0}-\alpha_{i}}\right]^{k} g(t) .
\end{aligned}
$$

Therefore, we see that, for any natural number $n$, the $n$th approximation solution is

$$
y^{n}(t)=\sum_{k=0}^{n}(-1)^{k} I_{0+}^{\alpha_{0}}\left[\sum_{i=1}^{m} a_{i}(t) I_{0+}^{\alpha_{0}-\alpha_{i}}\right]^{k} g(t)
$$

and

$$
y(t)=\lim _{n \rightarrow \infty} y^{n}(t)=\sum_{k=0}^{\infty}(-1)^{k} I_{0+}^{\alpha_{0}}\left[\sum_{i=1}^{m} a_{i}(t) I_{0+}^{\alpha_{0}-\alpha_{i}}\right]^{k} g(t),
$$

which yields $y(t) \in C^{\alpha_{0}, n_{0}-1}[0, T]$.

Theorem 3.2 Let $n_{0}-1<\alpha_{0} \leq n_{0}$ and $n_{0}=n_{1}$. Assume that $a_{i} \in C_{r}^{1}[0, T], D_{0+}^{n_{0}-\alpha_{0}} a_{i} \in$ $C[0, T], i=1, \ldots, m$ for $\gamma$ such that $0<\gamma=n_{0}-\alpha_{0}<1$ and $g(t) \in C[0, T]$. Moreover, assume that the case 1 holds. Then the initial value problem (1) and (2) has the unique solution $y(t) \in C^{\alpha_{0}, n_{0}-1}[0, T] \cap C_{\gamma}^{n_{0}}[0, T]$ and it is represented by

$$
y(t)=\sum_{j=0}^{n_{0}-1} b_{j} y_{j}(t)+\sum_{k=0}^{\infty}(-1)^{k} I_{0+}^{\alpha_{0}}\left[\sum_{i=1}^{m} a_{i}(t) I_{0+}^{\alpha_{0}-\alpha_{i}}\right]^{k} g(t),
$$

where $y_{j}(t)$ is the canonical fundamental system of solution to the corresponding homogeneous equation

$$
{ }^{c} D_{0+}^{\alpha_{0}} y(t)+\sum_{i=1}^{m} a_{i}(t)^{c} D_{0+}^{\alpha_{i}} y(t)=0, \quad t \in[0, T]
$$

which is obtained from Lemma 3.2.

Proof The proof follows from Theorem 3.1 and the linearity of the initial value problem (1) and (2).

Corollary 3.2.1 Let $n_{0}-1<\alpha_{0}<n_{0}, n_{0}>n_{1}, g(t) \in C[0, T]$ and $a_{i} \in C[0, T], i=1, \ldots m$. Assume that the case 1 holds true. Then the initial value problem (1) and (2) has the unique 
solution $y(t) \in C^{\alpha_{0}, n_{0}-1}[0, T]$ and it is represented as

$$
y(t)=\sum_{j=0}^{n_{0}-1} b_{j} y_{j}(t)+\sum_{k=0}^{\infty}(-1)^{k} I_{0+}^{\alpha_{0}}\left[\sum_{i=1}^{m} a_{i}(t) I_{0+}^{\alpha_{0}-\alpha_{i}}\right]^{k} g(t),
$$

where $y_{j}(t)$ is obtained from Lemma 3.3.

Corollary 3.2.2 Let $n_{0}-1<\alpha_{0} \leq n_{0}$ and $n_{0}=n_{1}$. Assume that $a_{i} \in C_{r}^{1}[0, T], D_{0+}^{n_{0}-\alpha_{0}} a_{i} \in$ $C[0, T], i=1, \ldots, m$ for any $\gamma$ such that $0<\gamma=n_{0}-\alpha_{0}<1$ and $g(t) \in C[0, T]$. Moreover, assume that the case 2 holds true. Then the initial value problem (1) and (2) has the unique solution $y(t) \in C^{\alpha_{0}, n_{0}-1}[0, T] \cap C_{\gamma}^{n_{0}}[0, T]$ and it is represented as

$$
y(t)=\sum_{j=0}^{n_{0}-1} b_{j} y_{j}(t)+\sum_{k=0}^{\infty}(-1)^{k} I_{0+}^{\alpha_{0}}\left[\sum_{i=1}^{m} a_{i}(t) I_{0+}^{\alpha_{0}-\alpha_{i}}\right]^{k} g(t)
$$

where $y_{j}(t)$ is obtained from Lemma 3.4.

Corollary 3.2.3 Let $n_{0}-1<\alpha_{0}<n_{0}, n_{0}>n_{1}, g(t) \in C[0, T]$ and $a_{i} \in C[0, T], i=1, \ldots, m$. Assume that the case 2 holds true. Then the initial value problem (1) and (2) has the unique solution $y(t) \in C^{\alpha_{0}, n_{0}-1}[0, T]$ and it is represented as

$$
y(t)=\sum_{j=0}^{n_{0}-1} b_{j} y_{j}(t)+\sum_{k=0}^{\infty}(-1)^{k} I_{0+}^{\alpha_{0}}\left[\sum_{i=1}^{m} a_{i}(t) I_{0+}^{\alpha_{0}-\alpha_{i}}\right]^{k} g(t),
$$

where $y_{j}(t)$ is obtained from Lemma 3.5 .

Corollary 3.2.4 Let $a_{i} \in C_{r}^{1}[0, T], i=1, \ldots$, m for $r$ such that $0<\gamma<\alpha_{0}-n_{0}+1$. Moreover, assume that the case 3 holds true. Then the initial value problem (1) and (2) has the unique solution $y(t) \in C^{\alpha_{0}, n_{0}-1}[0, T] \cap C_{\gamma}^{n_{0}}[0, T]$ and it is represented as

$$
y(t)=\sum_{j=0}^{n_{0}-1} b_{j} y_{j}(t)+\sum_{k=0}^{\infty}(-1)^{k} I_{0+}^{\alpha_{0}}\left[\sum_{i=1}^{m} a_{i}(t) I_{0+}^{\alpha_{0}-\alpha_{i}}\right]^{k} g(t)
$$

where $y_{j}(t)$ is obtained from Lemma 3.6.

\section{Examples}

Example 1 We consider the fractional differential equation with continuous variable coefficients as

$$
\left\{\begin{array}{l}
{ }^{c} D_{0+}^{0.8} y(t)+t^{s} \cdot{ }^{c} D_{0+}^{0.3} y(t)=t^{\beta} \\
\left.y(t)\right|_{t=+0}=0 .
\end{array}\right.
$$

Then we see that Eq. (29) satisfy the conditions of Theorem 3.1. We have

$$
y(t)=\sum_{k=0}^{\infty}(-1)^{k} I_{0+}^{0.8}\left(t^{k s} I_{0+}^{0.5 k} t^{\beta}\right)=I_{0+}^{0.8}\left(t^{\beta}\right)+\sum_{k=1}^{\infty}(-1)^{k+1} I_{0+}^{0.8}\left(t^{(k+1) s} I_{0+}^{0.5(k+1)} t^{\beta}\right)
$$




$$
\begin{aligned}
& =I_{0+}^{0.8}\left(t^{\beta}\right)-\frac{1}{\Gamma(0.8)} \int_{0}^{t}(t-z)^{-0.2} \sum_{k=0}^{\infty}(-1)^{k} z^{(k+1) s} I_{0+}^{0.5(k+1)} z^{\beta} d z \\
& =\frac{\Gamma(\beta+1)}{\Gamma(\beta+1.8)} t^{\beta+0.8}-\frac{\Gamma(\beta+1)}{\Gamma(0.8)} \int_{0}^{t}(t-v)^{-0.2} v^{s+\beta+0.5} E_{0.5, \beta+1.5}\left(-v^{s+0.5}\right) d v .
\end{aligned}
$$

Thai is, the solution of Example 1 is expressed as

$$
y(t)=\frac{\Gamma(\beta+1)}{\Gamma(\beta+1.8)} t^{\beta+0.8}-\frac{\Gamma(\beta+1)}{\Gamma(0.8)} \int_{0}^{t}(t-v)^{-0.2} v^{s+\beta+0.5} E_{0.5, \beta+1.5}\left(-v^{s+0.5}\right) d v .
$$

Example 2 We discuss the linear inhomogeneous fractional differential equation with continuous variable coefficients as

$$
\left\{\begin{array}{l}
{ }^{c} D_{0+}^{1.5} y(t)+t^{0.3} \cdot{ }^{c} D_{0+}^{0.8} y(t)=g(t), \\
\left.D^{k} y(t)\right|_{t=+0}=0, \quad k=0,1,
\end{array}\right.
$$

where $g(t)=\frac{2}{\Gamma(1.5)} t^{0.5}+\frac{2}{\Gamma(2.2)} t^{1.5}$. The exact solution of Example 2 is $y(t)=t^{2}$. We have $\alpha_{0}=1.5, m=1, \alpha_{1}=0.8$,

$$
\begin{aligned}
y(t) & =\sum_{k=0}^{\infty}(-1)^{k} I_{0+}^{1.5}\left(t^{0.3 k} I_{0+}^{0.7 k} g(t)\right)=I_{0+}^{1.5}(g(t))+\sum_{k=0}^{\infty}(-1)^{k+1} I_{0+}^{1.5}\left(t^{0.3(k+1)} I_{0+}^{0.7(k+1)} g(t)\right) \\
& =I_{0+}^{1.5}(g(t))-\frac{1}{\Gamma(1.5)} \int_{0}^{t}(t-z)^{0.5} \sum_{k=0}^{\infty}(-1)^{k} z^{0.3(k+1)} I_{0+}^{0.7(k+1)} g(z) d z .
\end{aligned}
$$

Also since

$$
\begin{aligned}
I_{0+}^{0.7(k+1)} g(z) & =\frac{2}{\Gamma(1.5)} I_{0+}^{0.7(k+1)} z^{0.5}+\frac{2}{\Gamma(2.2)} I_{0+}^{0.7(k+1)} z^{1.5} \\
& =\frac{2}{\Gamma(0.7(k+1)+1.5)} z^{0.5+0.7(k+1)}+\frac{2}{\Gamma(2.2)} \frac{\Gamma(2.5)}{\Gamma(0.7(k+1)+2.5)} z^{0.7(k+1)+1.5}
\end{aligned}
$$

and

$$
\begin{aligned}
I_{0+}^{1.5}(g(t)) & =\frac{2}{\Gamma(1.5)} I_{0+}^{1.5} t^{0.5}+\frac{2}{\Gamma(2.2)} I_{0+}^{1.5} t^{1.5}=\frac{2}{\Gamma(1.5)} \frac{\Gamma(1.5)}{\Gamma(3)} t^{2}+\frac{2}{\Gamma(2.2)} \frac{\Gamma(2.5)}{\Gamma(4)} t^{3} \\
& =t^{2}+\frac{\Gamma(2.5)}{3 \Gamma(2.2)} t^{3}
\end{aligned}
$$

are satisfied, we have

$$
\begin{aligned}
y(t)= & I_{0+}^{1.5}(g(t))-\frac{1}{\Gamma(1.5)} \int_{0}^{t}(t-z)^{0.5} \sum_{k=0}^{\infty}(-1)^{k} z^{0.3(k+1)} I_{0+}^{0.7(k+1)} g(z) d z \\
= & t^{2}+\frac{\Gamma(1.5)}{2 \Gamma(2.2)} t^{3}-\frac{2}{\Gamma(1.5)} \int_{0}^{t}(t-z)^{0.5} z^{1.5} E_{0.7,2.2}(-z) d z \\
& -\frac{3}{\Gamma(2.2)} \int_{0}^{t}(t-z)^{0.5} z^{2.5} E_{0.7,3.2}(-z) d z,
\end{aligned}
$$

where $E_{\alpha, \beta}(x)$ is the Mittag-Leffler function. 
Figure 1 The error between the exact solution and series-representation solution

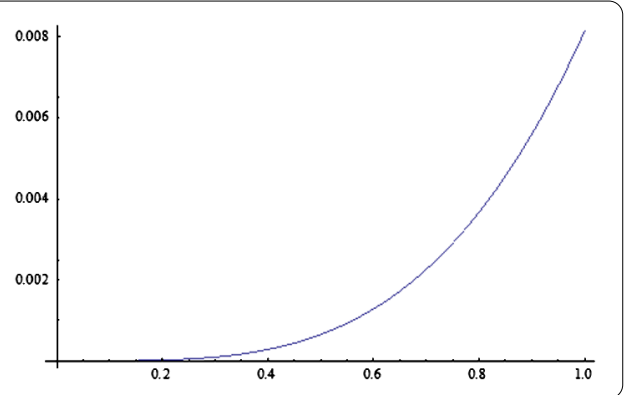

Therefore the solution expression $y(x)$ of the proposed problem is obtained as the above equation. The error between the exact solution and series-representation solution is

$$
\begin{aligned}
\Delta y= & \frac{\Gamma(1.5)}{2 \Gamma(2.2)} t^{3}-\frac{2}{\Gamma(1.5)} \int_{0}^{t}(t-z)^{0.5} z^{1.5} E_{0.7,2.2}(-z) d z \\
& -\frac{3}{\Gamma(2.2)} \int_{0}^{t}(t-z)^{0.5} z^{2.5} E_{0.7,3.2}(-z) d z .
\end{aligned}
$$

We can know that the error value is equal to almost zero. The error graph is shown in Fig. 1.

\section{Conclusion}

In this paper, we have obtained series-representations for the solution of initial value problems of linear inhomogeneous fractional differential equations with continuous variable coefficients and inhomogeneous initial conditions. We have proved that the solution of the problem is determined by adding the solution of the inhomogeneous differential equations with the homogeneous initial conditions to the linear combination of the canonical fundamental system of solution for the corresponding homogeneous fractional differential equation and the inhomogeneous initial values. The effectiveness of the proposed theory is illustrated with two examples. The representation of the solution for a multi-term fractional differential equation under general conditions will be the object of our future research.

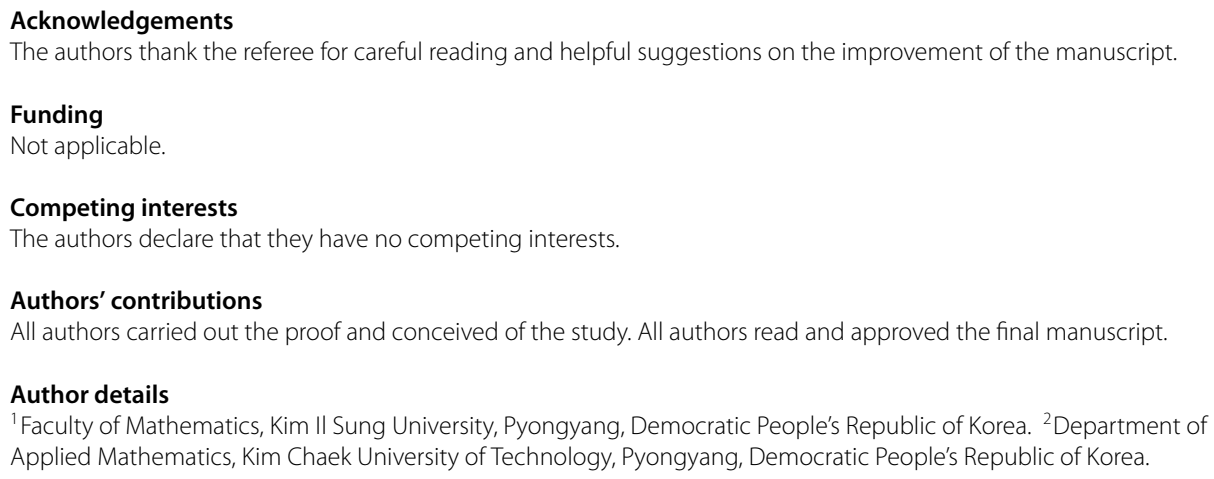

\section{Publisher's Note}

Springer Nature remains neutral with regard to jurisdictional claims in published maps and institutional affiliations. 


\section{References}

1. Engheta, N.: On fractional calculus and fractional multipoles in electromagnetism. IEEE Trans. Antennas Propag. 44, 554-566 (1996)

2. Ahmadian, A., Salahshour, S., Seng Chan, C.: Fractional differential systems: a fuzzy solution based on operational matrix of shifted Chebyshev polynomials and its applications. IEEE Trans. Fuzzy Syst. 25(1), $218-236$ (2016)

3. Ortigueira, M.D., Machado, J.A.T.: Fractional signal processing and applications. Signal Process. 83, 2285-2286 (2003)

4. Dehghan, M., Hamedi, E.A., Khosravian-Arab, H.: A numerical scheme for the solution of a class of fractional variational and optimal control problems using the modified Jacobi polynomials. J. Vib. Control 22(6), 1547-1559 (2016)

5. Bai, Z., Sun, S., Chen, Y., Bai, Z., Sun, S., Chen, Y.: The existence and uniqueness of a class of fractional differential equations. Abstr. Appl. Anal. 2014, Article ID 486040 (2014)

6. Lv, L., Wang, J., Wei, W.: Existence and uniqueness results for fractional differential equations with boundary value conditions. Opusc. Math. 31, 629-643 (2011)

7. Mahto, L., Abbas, S.: Existence and uniqueness of solution of Caputo fractional differential equations. AlP Conf. Proc. 1479, 896-899 (2012)

8. Tariboon, J., Sitthiwirattham, T., Ntouyas, S.K.: Boundary value problems for a new class of three-point nonlocal Riemann-Liouville integral boundary conditions. Adv. Differ. Equ. 2013, 213 (2013)

9. Liu, S.L., Li, H.L., Dai, Q., Liu, J.P.: Existence and uniqueness results for nonlocal integral boundary value problems for fractional differential equations. Adv. Differ. Equ. 2016, 122 (2016)

10. Badr, M., Yazdani, A., Jafari, H.: Stability of a finite volume element method for the time-fractional advection-diffusion equation. Numer. Methods Partial Differ. Equ. 34(5), 1459-1471 (2018)

11. Doha, E.H., Bhrawy, A.H., Baleanu, D., Ezz-Eldien, S.S.: On shifted Jacobi spectral approximations for solving fractional differential equations. Appl. Math. Comput. 219, 8042-8056 (2013)

12. Sin, K., Chen, M., Choi, H., Ri, K.: Fractional Jacobi operational matrix for solving fuzzy fractional differential equation. J. Intell. Fuzzy Syst. 33, 1041-1052 (2017)

13. Diethelm, K., Ford, N.J., Freed, A.D.: A predictor corrector approach for the numerical solution of fractional differential equations. Nonlinear Dyn. 29, 3-22 (2002)

14. Doha, E.H., Bhrawy, A.H., Ezz-Eldien, S.S.: A Chebyshev spectral method based on operational matrix for initial and boundary value problems of fractional order. Comput. Math. Appl. 62, 2364-2373 (2011)

15. Mazandarani, M., Vahidian Kamyad, A.: Modified fractional Euler method for solving fuzzy fractional initial value problem. Commun. Nonlinear Sci. Numer. Simul. 18, 12-21 (2013)

16. Sadeghi Roshan, S., Jafari, H., Baleanu, D.: Solving FDEs with Caputo-Fabrizio derivative by operational matrix based on Genocchi polynomials. Math. Methods Appl. Sci. 41(18), 9134-9141 (2018)

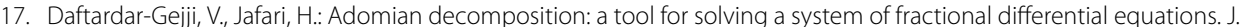
Math. Anal. Appl. 301, 508-518 (2005)

18. Jafari, H.: Numerical solution of time-fractional Klein-Gordon equation by using the decomposition methods. J. Comput. Nonlinear Dyn. 11(4), 041015 (2016)

19. Jafari, H., Momani, S.: Solving fractional diffusion and wave equations by modified homotopy perturbation method. Phys. Lett. A 370, 388-396 (2007)

20. Das, S.: Analytical solution of a fractional diffusion equation by variational iteration method. Comput. Math. Appl. 57, 483-487 (2009)

21. Firoozjaee, M.A., Jafari, H., Lia, A., Baleanu, D.: Numerical approach of Fokker-Planck equation with Caputo-Fabrizio fractional derivative using Ritz approximation. J. Comput. Appl. Math. 339, 367-373 (2018)

22. Hu, Y., Luo, Y., Lua, Z:: Analytical solution of the linear fractional differential equation by Adomian decomposition method. J. Comput. Appl. Math. 215, 220-229 (2008)

23. Bonilla, B., Rivero, M., Trujillo, J.J.: On system of linear fractional differential equation with constant coefficients. Appl. Math. Comput. 181, 68-78 (2007)

24. Kilbas, A.A., Rivero, M., Rodríguez-Germá, L., Trujillo, J.J.: $\alpha$-Analytic solutions of some linear fractional differential equations with variable coefficients. Appl. Math. Comput. 187, 239-249 (2007)

25. Umarov, S.R., Saidamatov, E.M.: A generalization of Duhamel's principle for differential equations of fractional order. Dokl. Math. 75(1), 94-96 (2007)

26. Cui, R., Ban, T.: An analytical solution for a fractional heat-like equation with variable coefficients. Therm. Sci. 21(4), 1759-1764 (2017)

27. Diethelm, K.: The Analysis of Fractional Differential Equations. Springer, Berlin (2010)

\section{Submit your manuscript to a SpringerOpen ${ }^{\circ}$ journal and benefit from:}

- Convenient online submission

- Rigorous peer review

- Open access: articles freely available online

- High visibility within the field

Retaining the copyright to your article

Submit your next manuscript at $\boldsymbol{s p r i n g e r o p e n . c o m ~}$ 